\title{
The effect of therapeutic temperature management on cell death and apoptosis in resuscitative attempts
}

\begin{abstract}
Cardiac arrests (CA) occurred in- and out-of-hospital is the plague of the modern day and therapeutic temperature management (TTM) is claimed to be a remedy against this. TTM is practised mainly in the treatment of adult cardiac arrest and neonatal hypoxic-ischemic encephalopathy (HIE). Neurologic injury is the most common cause of death in patients with OHCA. TTM is commonly recommended for survivors of cardiac arrest as a common final pathway of various mechanisms to alleviate the neurologic injury. TTM can be separated into three phases: induction, maintenance and rewarming; and each phase produces several changes in normal physiology. The induction of mild to moderate TTM to a target temperature 32 to $34^{\circ} \mathrm{C}$ in the initial hours after cardiac arrest improves the neurologic outcome of resuscitated patients. TTM acts in many fields by affecting several injury mechanisms concurrently to reduce death rate of the brain cell. This article is written to review the current literature to summarize data regarding cellular injury following CA and effects attributed to the procedure of TTM in the emergency setting.
\end{abstract}

Keywords: cardiac arrest, cell death, apoptosis, targeted temperature management, therapeutic hypothermia
Volume 5 Issue I - 2018

\author{
Ozgur Karcioglu \\ Department of emergency medicine, University of Health \\ Sciences, Istanbul Education and Research Hospital,Turkey
}

\author{
Correspondence: Ozgur Karcioglu, Research Hospital, \\ Emergency Department, University of Health Sciences, Istanbul \\ Training, Turkey, Tel 00905055252399 ,
}

Email okarcioglu@gmail.com

Received: November 0I, 2017 | Published: January 08, 2018
Abbreviations: CA, cardiac arrests; TTM, therapeutic temperature management; HIE, hypoxic ischemic encephalopathy; TLR, toll like receptor; ATP, adenosine tri phosphate

\section{Introduction}

Therapeutic temperature management (TTM) is launched to be a solution against high mortality rate of in- or out-of-hospital cardiac arrests (CA). The technique is mostly undertaken in the treatment of adult CA and neonatal hypoxic-ischemic encephalopathy (HIE). It is also performed to provide neuroprotection during certain types of surgery and after serious events that threatens the brain.

TTM has been historically classified into: mild $\left(34.5-36.5^{\circ} \mathrm{C}\right)$, moderate $\left(34.5-32^{\circ} \mathrm{C}\right)$, marked $\left(28-32^{\circ} \mathrm{C}\right)$ and profound hypothermia $\left(<28^{\circ} \mathrm{C}\right) .^{1,2}$ The technique can be separated into three phases: induction, maintenance and rewarming; and each phase produces several changes in normal physiology. Very recent literature data pointed out that in real-world practices without a strictly controlled environment, TTM can improve survival and favorable neurological outcome in post-CA patients independent of initial rhythm. ${ }^{3}$ Furthermore, international consensus groups and committees recommended TTM for adults with $\mathrm{CA}$ with an initial shockable rhythm at a constant temperature between $32^{\circ} \mathrm{C}$ and $36^{\circ} \mathrm{C}$ for at least 24 hours. ${ }^{4}$ In the post-resuscitative period, TTM is thought to mitigate neurologic reperfusion injury by decreasing cerebral oxygen consumption and biochemical damage. 5 TTM was postulated to offer an extended therapeutic window to restore the integrity of circulation, with the brain maintained in a protective, hypo-metabolic state. Prolonged, moderate cerebral hypothermia initiated within a few hours after severe HIE and maintained until resolution of the acute phase of delayed cell death is reported to reduce acute brain injury in term infants and in adults after CA. ${ }^{6}$ The specific mechanisms of hypothermic neuro protection are not yet clear, partly because TTM suppresses a myriad of potential injurious factors. This article is written to review the current literature to summarize data regarding cellular injury following CA and effects attributed to the procedure of TTM in the emergency setting.

\section{Cardiac arrest, cell death and TTM}

In the course of ischaemic brain injury, necrotic cell death or apoptosis can ensue in the brain cells. ${ }^{7}$ More specifically, there are three phases of brain injury in patients with CA. ${ }^{8,9}$ The first phase is intra-arrest ischemic injury due to absence of blood flow. Failure of energy, ischemic depolarization of cell membranes, release of excitatory amino acids, and cytosolic calcium overload are noted in this phase. Irreversible injury can ensue should prolonged ischemia takes place. The second phase comprises immediate reperfusion injury seen after successful resuscitation. In this phase, the resumption of oxidative phosphorylation can cause production of reactive oxygen species, calcium overload and permeability transition in the mitochondria, which trigger cell death signaling. The third phase consists of post-reperfusion injury encountered late after resuscitation. Secondary calcium overload in the neural cells, pathologic protease activation, and altered gene expression and inflammation can occur and can last for several days. ${ }^{10}$ Proper resuscitation is essential and effective in restoring energy stores in the brain tissue. A diminution in cerebral metabolism at low temperatures may enlighten why hypothermia can protect brain cells in ischaemic conditions. It has been shown empirically that brain glucose turnover is reduced by $5 \%$ for every degree the temperature is lowered. ${ }^{11}$ Assuming a direct correlation between metabolism and neuroprotection, cooling to $33^{\circ} \mathrm{C}$, for example, should end up with a one-fifth reduction in cellular damage. ${ }^{12,13}$

Mild hypothermia $\left(32^{\circ} \mathrm{C}\right.$ to $\left.34^{\circ} \mathrm{C}\right)$ induced via TTM technique for brain protection was performed for a long time in a number of surgical procedures and circulatory arrest states. The synthesis, release and uptake of certain catecholamines and neurotransmitters are inhibited by TTM..$^{14,15}$ Inhibition of glutamate and dopamine, for 
example, prevents probable damage to the tissues. ${ }^{16,17}$ Blood-brain barrier is reportedly maintained via TTM ${ }^{18}$ TTM also helps protection of adenosine triphosphate (ATP) stores. ${ }^{19}$ TTM acts in many fields by affecting several injury mechanisms concurrently ${ }^{20}$ to reduce death rate of the brain cell. Apoptosis can be halted by TTM affecting both caspase-dependent and caspase-independent cellular mechanisms. In addition, certain cold shock proteins can augment cell survival by inhibiting apoptosis specifically during cooling. ${ }^{12}$ The accumulation and release of glutamate are decreased, and thus effects on glutamate receptors can reduce hazards of calcium influx into cells. ${ }^{7}$ Hypothermia can inhibit inflammatory responses to ischaemia, such that formation of oxygen free radicals, reactive nitrogen compounds, cytokines and matrix metalloproteases. ${ }^{21,22}$

\section{Inflammatory and immune response}

Post-arrest patients have a modest systemic inflammatory response compared to healthy controls, associated with lower HLA-DR expression and attenuated immune response to Gramnegative and Gram-positive antigens. ${ }^{23}$ Inhibition of the exaggerated systemic inflammatory response syndrome is thought to be one of the mechanisms through which TTM can mitigate the harmful effects of ischemia-reperfusion. In a pig model of CA, TTM reduced expression of pro-inflammatory cytokines within the brain. ${ }^{24}$ Also in other models of hyper-inflammatory conditions, TTM reduced organ failure associated with a decrease of the inflammatory response. ${ }^{25-29}$ Cooling modulates production of a range of inflammatory mediators implicated in secondary brain injury. Chen et al., ${ }^{30}$ assayed levels of six of these in a swine model of lethal haemorrhage, applying cardiopulmonary bypass with a hyperkalaemic perfusion fluid after 30 minutes of haemorrhage. Cooled animals exhibited decreased serum levels of pro-inflammatory IL-6, compared to controls. Levels of a protective heat shock protein, HSP-70, and an anti-inflammatory cytokine, IL-10, were found to be elevated in the cooled animals. Other inflammatory cytokines released by activated microglia and astrocytes include IL-1 $\beta$, IL-18 and TNF- $\alpha$, and modulation of this response is observed in the process of TTM. ${ }^{31}$

Beurskens et al., ${ }^{23}$ conducted a cohort study to enlighten the impact of TTM on immune response after CA. They reported that CA patients maintained in mild hypothermia (at $36^{\circ} \mathrm{C}$ ) had higher plasma cytokines levels than that of healthy controls, which was not apparent in patients kept at $33^{\circ} \mathrm{C}$. Immune response to toll-like receptor (TLR) ligands in patients after $\mathrm{CA}$ was generally reduced and associated with lower HLA-DR expression. Patients kept at $33^{\circ} \mathrm{C}$ had preserved ability of immune cells to respond to lipopolysaccharide and lipoteicoic acid compared to patients kept at $36^{\circ} \mathrm{C}$. These differences disappeared over time. HLA-DR expression did not differ between $33^{\circ} \mathrm{C}$ and $36^{\circ} \mathrm{C}$. Patients with a body temperature of $33^{\circ} \mathrm{C}$ did not differ from patients with a body temperature of $36^{\circ} \mathrm{C}$, suggesting TTM does not affect immune response in patients with CA.

\section{Some animals studies focused on cell survival and TTM}

Hypothermia was shown to potentiate the activation of various molecular mechanisms which are involved in cell survival pathways. This promising finding occurred specifically in the pre-synaptic mossy fibres in the stratum lucidum of the hippocampus, which indicates an important role of hypothermia in cell survival. ${ }^{32}$ One of the mechanisms that postulate the protective effect of hypothermia against hazards of head injury is attenuation of the expression of various pro-inflammatory cytokines taking part in the pathogenesis of injury such that IL-1 $\beta$, IL2, IL6 and TGF $\beta-2 .{ }^{33,34}$ A study showed that IL-10 production was reduced in rat microglia cultures under hypothermic conditions, suggesting that the neuroprotective effects of hypothermia may paradoxically involve the suppression of antiinflammatory cytokines as well. ${ }^{35}$ Cooling also suppressed the $\mathrm{X}$-linked inhibitor of apoptosis protein cleavage thus promoting its binding onto caspases and inhibiting the protease activity. ${ }^{36}$ Using an ischaemia-reperfusion model, Kobayashi et al., ${ }^{37}$ screened 24,000 genes using high density oligonucleotide microarray and found that the expression of 33 genes were temperature-dependent. Hypothermia has an impact on a range of protein kinases involved in cellular transcription regulation. This leads to a diminution of necrosis, and a secondary reduction in the inflammation that would otherwise contribute to the cycle of secondary injury. In a recent study Moffatt et al., ${ }^{38}$ conducted a systematic review and culminated data on 327 animals cooled to $\leq 20^{\circ} \mathrm{C}$ after hemorrhagic shock in these trials. Evidence regarding profound hypothermia suggests that this form of resuscitation modality could be beneficial to the patients with hemorrhagic shock. Animal studies, as a whole, demonstrate a clear benefit of the use of TTM in the management of cell survival in the context of head injury. Although the exact mechanisms are still unknown, it is apparent that hypothermia exerts its effects in many pathways of injury in brain trauma. ${ }^{39}$

\section{Conclusion}

TTM acts in many fields by affecting several injury mechanisms concurrently to reduce death rate of the brain cell. Although the exact mechanisms are still unknown, it is apparent that hypothermia exerts its effects in many pathways of injury in both patients with head trauma and those resuscitated from arrest situations.

\section{Acknowledgements}

None.

\section{Conflict of interest}

The author declares no conflict of interest.

\section{References}

1. Tisherman SA, Rodriguez A, Safar P. Therapeutic hypothermia in traumatology. Surg Clin North Am. 1999;79(6):1269-1289.

2. Andresen M, Gazmuri JT, Marín A, et al. Therapeutic hypothermia for acute brain injuries. Scand J Trauma Resusc Emerg Med. 2015;23:42.

3. Srivilaithon W, Muengtaweepongsa S. The Outcomes of Targeted Temperature Management After Cardiac Arrest at Emergency Department: A Real-World Experience in a Developing Country. Ther Hypothermia Temp Manag. 2017;7(1):24-29.

4. Donnino MW, Andersen LW, Berg KM, et al. Temperature Management After Cardiac Arrest: An Advisory Statement by the Advanced Life Support Task Force of the International Liaison Committee on Resuscitation and the American Heart Association Emergency Cardiovascular Care Committee and the Council on Cardiopulmonary, Critical Care, Perioperative and Resuscitation. Resuscitation. 2015;98:97-104.

5. Hypothermia after Cardiac Arrest Study Group. Mild therapeutic hypothermia to improve the neurologic outcome after cardiacarrest. N Engl J Med. 2002;346(8):549-556.

6. Drury PP, Gunn ER, Bennet L, et al. Mechanisms of hypothermic neuroprotection. Clin Perinatol. 2014;41(1):161-175. 
7. Dietrichs ES, Dietrichs E. Neuroprotective effects of hypothermia. Tidsskr Nor Laegeforen. 2015;135(18):1646-1651.

8. Perman SM, Goyal M, Neumar RW, et al. Clinical applications of targeted temperature management. Chest. 2014;145(2):386-393.

9. Polderman KH. Mechanisms of action, physiological effects, and complications of hypothermia. Crit Care Med. 2009;37(7 Suppl):S186S202.

10. Fukuda T. Targeted temperature management for adult out-of-hospital cardiac arrest: current concepts and clinical applications. J Intensive Care. 2016;4:30.

11. Laptook AR, Corbett RJT, Sterett R, et al. Quantitative relationship between brain temperature and energy utilization rate measured in vivo using $31 \mathrm{P}$ and $1 \mathrm{H}$ magnetic resonance spectroscopy. Pediatr Res. 1995;38:919-925.

12. Yenari MA, Han HS. Neuroprotective mechanisms of hypothermia in brain ischaemia. Nat Rev Neurosci. 2012;13:267-278.

13. Urrea C, Danton GH, Bramlett HM, et al. The beneficial effect of mild hypothermia in a rat model of repeated thromboembolic insults. Acto Neuropathol. 2004;107(5):413-420.

14. Sun S, Tang W, Song F, et al. The effects of epinephrine on outcomes of normothermic and therapeutic hypothermic cardiopulmonary resuscitation. Crit Care Med. 2010;38(11):2175-2180.

15. Szelenyi M, Laszlo Z, Szabo Z, et al. Hypothermia down-regulates the LPS induced norepinephrine (NE) release in ischaemic human heart cells. Brain Res Bull. 2012;87(1):67-73.

16. D’Cruz BJ, Fertig KC, Filiano AJ, et al. Hypothermic reperfusion after cardiac arrest augments brain-derived neurotrophic factor activation. $J$ Cerebr Blood Flow Metab. 2002;22(7):843-851.

17. Hachimi-Idrissi S, Van Hemelrijck A, Michotte A, et al. Postischemic mild hypothermia reduces neurotransmitter release and astroglial cell proliferation during reperfusion after asphyxial cardiac arrest in rats. Brain Res. 2004;1019(1-2):217-225.

18. Baumann E, Preston E, Slinn J, et al. Postischemic hypothermia attenuates loss of the vascular basement membrane proteins, agrin and SPARC, and the blood-brain barrier disruption after global cerebral ischemia. Brain Res. 2009;1269:185-197.

19. McCullough JN, Zhang N, Reich DL, et al. Cerebral metabolic suppression during hypothermic circulatory arrest in humans. Ann Thorac Surg. 1999;67(6):1895-1899.

20. Holzer M. Targeted temperature management for comatose survivors of cardiac arrest. The New Engl J Med. 2010;363(13):1256-1264.

21. $\mathrm{Wu} \mathrm{T}-\mathrm{C}$, Grotta JC. Hypothermia for acute ischaemic stroke. Lancet Neurol. 2013;12(3):275-284.

22. Ceulemans A-G, Zgavc T, Kooijman R, et al. The dual role of the neuroinflammatory response after ischemic stroke: modulatory effects of hypothermia. J Neuroinflammation. 2010;7:74.

23. Beurskens CJ, Horn J, de Boer AM, et al. Cardiac arrest patients have an impaired immune response, which is not influenced by induced hypothermia. Crit Care. 2014;18(4):R162.
24. Meybohm P, Gruenewald M, Zacharowski KD, et al. Mild hypothermia alone or in combination with anesthetic post-conditioning reduces expression of inflammatory cytokines in the cerebral cortex of pigs after cardiopulmonary resuscitation. Crit Care. 2010;14(1):R21.

25. Beurskens CJ, Aslami H, Kuipers MT, et al. Induced hypothermia is protective in a rat model of pneumococcal pneumonia associated with increased adenosine triphosphate availability and turnover. Crit Care Med. 2012;40(3):919-926.

26. Taniguchi T, Kanakura H, Takemoto Y, et al. Effects of hypothermia on mortality and inflammatory responses to endotoxin-induced shock in rats. Clin Diagn Lab Immunol. 2003;10(5):940-943.

27. Chu SJ, Perng WC, Hung CM, et al. Effects of various body temperatures after lipopolysaccharide-induced lung injury in rats. Chest. 2005;128(1):327-336.

28. Chin JY, Koh Y, Kim MJ, et al. The effects of hypothermia on endotoxinprimed lung. Anesth Analg. 2007;104(5):1171-1178.

29. Kira S, Daa T, Kashima K, et al. Mild hypothermia reduces expression of intercellular adhesion molecule-1 (ICAM-1) and the accumulation of neutrophils after acid-induced lung injury in the rat. Acta Anaesthesiol Scand. 2005;49(3):351-359.

30. Chen Z, Chen $\mathrm{H}$, Rhee P, et al. Induction of profound hypothermia modulates the immune/inflammatory response in a swine model of lethalhemorrhage. Resuscitation. 2005;66(2):209-216.

31. Morino T, Ogata T, Takeba J, et al. Microglia inhibition is a target of mild hypothermic treatment after the spinal cord injury. Spinal Cord. 2008;46(6):425-431.

32. Atkins CM, Oliva AA, Alonso OF, et al. Hypothermia treatmen potentiates ERK1/2 activation after traumatic brain injury. Eur $J$ Neurosci. 2007;26(4):810-819.

33. Kinoshita K, Chatzipanteli K, Vitarbo E, et al. Interleukin-1beta messenger ribonucleic acid and protein levels after fluid-percussion braininjury in rats: importance of injury severity and brain temperature. Neurosurgery. 2002;51(1):195-203.

34. Truettner JS, Alonso OF, Dietrich WD. Influence of therapeutic hypothermia on matrix metalloproteinase activity after traumatic brain injury in rats. J Cerebr Blood Flow Metabol. 2005;25(11):1505-1516.

35. Matsui T, Kakeda T. IL-10 production is reduced by hypothermia but augmented by hyperthermia in rat microglia. $J$ Neurotrauma. 2008;25(6):709-715.

36. Qiu L. Hypothermia in Traumatic Brain Injury-A Literature Review. $J$ Intens Care Soc. 2011;12(3):201-214.

37. Kobayashi MS, Asai S, Ishikawa K, et al. Global profiling of influence of intra-ischemic brain temperature on gene expression in rat brain. Brain Research Rev. 2008;58(1):171-191.

38. Moffatt SE, Mitchell SJB, Walke JL. Deep and profound hypothermia in haemorrhagic shock, friend or foe? A systematic review. J R Army Med Corps. 2018;164(3):191-196.

39. Karcioglu O. Therapeutic Hypothermia in Trauma Management: New Tricks for an Old Dog? Emerg Med Open J. 2017;3(2):30-37. 